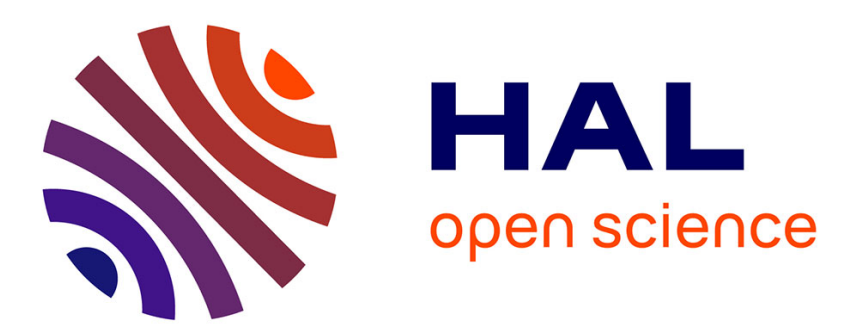

\title{
Quels parallèles entre les représentations du parler plurilingue et celles du parler pluristyle chez les enfants?
}

Laurence Buson, Jacqueline Billiez

\section{To cite this version:}

Laurence Buson, Jacqueline Billiez. Quels parallèles entre les représentations du parler plurilingue et celles du parler pluristyle chez les enfants?. Maria Iliescu; Paul Danler; Heidi Siller. Actes du XXVe Congrès International de Linguistique et de Philologie Romanes, De Gruyter, pp.4-47-4-56, 2010, 9783110231922. 10.1515/9783110231922.4-47 . hal-03549626

\section{HAL Id: hal-03549626 \\ https://hal.science/hal-03549626}

Submitted on 14 Feb 2022

HAL is a multi-disciplinary open access archive for the deposit and dissemination of scientific research documents, whether they are published or not. The documents may come from teaching and research institutions in France or abroad, or from public or private research centers.
L'archive ouverte pluridisciplinaire HAL, est destinée au dépôt et à la diffusion de documents scientifiques de niveau recherche, publiés ou non, émanant des établissements d'enseignement et de recherche français ou étrangers, des laboratoires publics ou privés. 


\title{
Version provisoire
}

\section{Laurence Buson / Jacqueline Billiez.}

\author{
Quels parallèles entre les représentations du parler plurilingue et celles du \\ parler pluristyle chez les enfants ?
}

Dans cet article, nous nous proposons d'apporter quelques éléments de réponses à deux séries de questions: est-il pertinent de faire un parallèle entre l'étude des parlers plurilingues et l'étude de ce que l'on pourrait appeler les parlers pluristyles? Et quels types de parallèles peut-on faire? Ces premières questions appellent des réponses générales concernant tout type de locuteur, qu'il soit adulte, adolescent ou enfant.

La deuxième série de questions à laquelle nous tenterons d'apporter des réponses concerne des enfants scolarisés en fin d'école primaire en France: trouve-t-on des traces, à la fois dans les usages et dans les discours sur la variation, d'intentions ou de conscience du locuteur enfant de modifier la relation interpersonnelle dans les choix et / ou alternances stylistiques, au même titre que dans les alternances codiques?

Nous allons nous référer principalement à des discours d'enfants qui ont entre 9 et 12 ans (fin de l'école primaire française), discours recueillis essentiellement à l'occasion de deux études, ayant des objets de recherche différents: d'une part la thèse de Sabatier (2004) et d'autre part celle de Buson (en cours). Ces deux sources nous permettent d'aborder conjointement les représentations du plurilinguisme chez des enfants bilingues voire plurilingues', et les représentations de la variation stylistique auprès d'enfants tout-venants.

Les discours sur lesquels nous allons fonder notre analyse ont été suscités, provoqués ou déclenchés de diverses manières (notamment un recueil de réactions à partir de matériaux sonores déclencheurs, et des entretiens dans une approche ethnographique) pour faire émerger des représentations, des catégorisations et des évaluations.

Au plan théorique, notre réflexion s'appuie sur les connaissances acquises sur le terrain du plurilinguisme migratoire à partir d'un ensemble de travaux menés par des chercheurs dans différents pays européens, aux Etats-Unis, au Canada, mais aussi à Grenoble depuis maintenant plus de trente ans (Billiez, 1997), travaux où l'on retrouve l'affirmation maintes fois avancée selon laquelle, par une sorte d'effet de loupe, l'étude du comportement du locuteur bilingue et du parler bilingue serait susceptible de grossir et donc d'éclairer et de faciliter l'approche de celui du monolingue pluristyle. Cette hypothèse, notamment

1 Ces enfants sont en contact régulier avec des locuteurs d'origine immigrée ou non immigrée du Maghreb, qu'il s'agisse de la famille (famille élargie ou nucléaire) ici et là-bas, des amis du quartier et de l'école, dans le cadre d'échanges langagiers directs ou médiatisés (télévision, téléphone, cassettes, etc.). 
développée dans l'ouvrage fondamental sur le bilinguisme que l'on doit à Lüdi / Py (2003) n'a jamais, à notre connaissance, réellement été prise au mot. C'est pourquoi nous avons tenté de la mettre à l'épreuve, à la fois des faits empiriques et des théories.

Dans cette contribution, des parallèles vont donc être envisagés sur deux plans: d'une part, celui du savoir plus ou moins savant (non totalement dégagé d'idéologie) à travers l'évolution des concepts, notions, conceptions du bi-plurilinguisme et des choix / alternances de langues, et / ou de styles; d'autre part, au plan de l'analyse d'extraits de corpus qui permettront de mettre en lumière les représentations que des enfants peuvent se faire de ces variations et de ces choix et / ou alternances stylistiques. On s'interrogera en fin de parcours sur les limites de ces comparaisons entre ce que nous avons nommé parler plurilingue et parler pluristyle.

\section{L'évolution des concepts}

Sans prétendre nous livrer à un panorama exhaustif, nous allons mettre en évidence quelques aspects convergents concernant l'évolution des conceptions des parlers plurilingues et pluristyles, évolution qui nous est apparue comme relativement similaire.

Tout d'abord, il est remarquable de constater que, pendant longtemps, l'alternance des langues a été abordée à travers une conception d'un bilinguisme parfait, équilibré, avec des choix de langues bien séparés et où le véritable bilingue pouvait à tout moment et en toutes circonstances s'exprimer dans l'une ou l'autre langue. Selon cette conception, le locuteur au bilinguisme inégal ne pouvait manifester dans son parler que des alternances d'incompétence dans l'une des deux langues. Aujourd'hui, ces types d'alternances sont plutôt envisagés dans leur complémentarité et les choix et alternances de langues le sont en termes de stratégies. On trouve en miroir une évolution convergente du côté de la variation stylistique, avec l'alternance des registres impliquant des variétés bien séparées, que certains locuteurs (ceux des classes supérieures en l'occurrence) seraient seuls en mesure d'utiliser. Le refus de qualifier de bilingue un locuteur qui n'a pas une maîtrise parfaite / complète des deux langues est donc à mettre en parallèle avec les conceptions de Bernstein (1975) du locuteur unistyle des classes populaires, enfermé dans un seul registre ("code restreint"). L'idée que tous les locuteurs, quels que soient la langue, le type de société et la place qu'ils occupent dans l'organisation sociale, sont pluristyles est avancée par Labov dans les années soixante-cinq dans le cadre de l'enquête à New York, alors que sur l'île de Martha's Vineyard (Labov, 1976), la conclusion de son étude allait dans le sens d'un locuteur unistyle.

En définitive, les notions de codes élaboré et restreint chez Bernstein seraient à rapprocher, du côté des travaux portant sur le bilinguisme, de la notion de bilinguisme soustractif, et de "semilinguisme". On a donc affaire à une conception séparative des langues et des niveaux de langues qui tiennent à des préjugés évoqués par Gadet / Varro (2006: 11):

Un vieux préjugé en a fait [du bilinguisme] un phénomène exceptionnel, lié à l'idée qu'il faut que la maîtrise des deux langues soit à la fois parfaite et équilibrée dans différentes fonctions, 
Quels parallèles entre les représentations du parler plurilingue et celles du parler pluristyles chez les enfants?

ce qui est rarement le cas. C'est là un effet d'une réflexion partant des langues et non de la personne et de son répertoire, et un effet d'une conception puriste /.../ que de penser les deux langues du bilingue comme clairement séparées, et le bilinguisme comme la juxtaposition de deux unilinguismes.

Ces préjugés ont la vie dure et circulent aussi dans le cadre scolaire: le fait de considérer que le monolinguisme est la norme, voire l'idéal, et le plurilinguisme l'exception, voire la déviance, est à rapprocher de l'idéologie normative scolaire selon laquelle "bien parler" signifie parler en langage soutenu, et non maîtriser une vaste gamme de styles permettant de s'adapter aux différents contextes communicatifs. La crainte chez les classes moyennes ou favorisées de la "contamination" langagière dans les environnements scolaires mixtes est donc à rapprocher de la méfiance qu'inspirent encore certains types de bilinguismes et de la conception des "conteneurs" (Gadet / Varro, 2006: 11) selon laquelle ce qui est gagné dans une langue est perdu pour l'autre.

Les recherches actuelles ont néanmoins permis aux représentations d'évoluer. Aujourd'hui, les conceptions savantes envisagent les ressources langagières et stylistiques au sein du répertoire communicatif de chaque locuteur. Alors que les parlers mixtes (avec l'idée de mélange, vu négativement au départ, de deux entités stabilisées) sont désormais envisagés comme des parlers bi-plurilingues, la conception en registres homogènes laisse maintenant place à ce que l'on pourrait appeler le parler pluristyle. Au final, les conceptions issues des recherches sur le plurilinguisme semblent précurseurs par rapport aux études dédiées à la variation stylistique: depuis Gumperz (1964), il est établi que le locuteur est actif et qu'il utilise de manière consciente ses ressources langagières comme une stratégie verbale. En revanche, il faut attendre les années quatre-vingt-dix pour voir apparaître l'idée de "speaker design approach" selon laquelle les choix stylistiques ne sont pas que des phénomènes réactifs (variation en réponse au degré de formalité de la situation ou à l'auditoire): "Under speaker design approaches, stylistic variation is viewed not as a reactive phenomenon but as a resource in the active creation, presentation, and re-creation of speaker identity." (Schilling-Estes, 2002: 388).

Notons enfin que le plurilinguisme est aujourd'hui appréhendé sous ses aspects dynamiques, faisant l'objet de multiples reconfigurations au cours de la biographie des locuteurs et étant pleinement ancré dans les usages, c'est-à-dire envisagé sous l'angle de la variabilité de ce que fait le locuteur dans des interactions situées. Sous cet abord dynamique, le retard est encore plus accusé du côté du parler pluristyle. De nombreuses questions restent en suspens: le répertoire stylistique du dit monolingue se reconfigure-t-il tout au long de la vie comme c'est le cas du répertoire plurilingue? Peut-on acquérir ou perdre un style? Autant de domaines de recherches encore aujourd'hui peu explorés.

Après ce tour d'horizon rapide de l'évolution des concepts, nous allons maintenant nous intéresser aux motivations et fonctions des choix de langues et de styles chez les enfants auprès desquels nous avons enquêté, de manière à mettre en évidence les éventuels parallèles entre code-switching et style-shifting (désormais CS et SS). 
2. Les choix / alternances stylistiques du monolingue font-ils écho aux choix / alternances de langues du biplurilingue chez les enfants?

Les enfants que nous avons observés et enregistrés utilisent-ils le SS comme une stratégie verbale, au même titre que les enfants biplurilingues dans le CS? Sur ces aspects, on peut mettre en perspective les propos de Gumperz (1989) et de Schilling-Estes (2002):

Une telle commutation [le CS] a d'importantes fonctions communicatives et comporte des significations qui, à bien des égards, sont semblables à celles des choix stylistiques dans les situations monolingues. (Gumperz, 1989: 111)

People utilize stylistic resources, not only to indicate relatively longstanding group affiliations and personal attributes but also to make temporary meanings in ongoing interaction, in other words, to accomplish various conversational purposes. (Schilling-Estes, 2002: 390)

Les chercheurs dans le domaine du plurilinguisme ont en effet, dans une perspective interactionnelle, relevé de multiples fonctions et motivations du CS. Nous en avons sélectionné quelques-unes que nous avons regroupées sous deux grandes catégories:

- d'une part, la dichotomie inclusion vs exclusion qui se traduit par la volonté de construire une image de soi, de revendiquer une identité, de se rapprocher d'une identité sociale valorisée, de s'identifier au moins temporairement à un groupe ou de s'en distancier,

- et, d'autre part, la relation dialogique entre convergence et divergence, suivant laquelle le locuteur agit pour modifier la relation interpersonnelle, pour entrer ou non en connivence avec son interlocuteur.

\subsection{Les fonctions / motivations du CS et du SS: inclusion $v s$ exclusion}

\subsubsection{Association d'une façon de parler et d'un groupe social (stéréotypé)}

Il est intéressant de noter que, dans les deux enquêtes sur lesquelles nous fondons notre analyse, des stéréotypes sociaux comme le style "racaille" ont été évoqués à de nombreuses reprises. Cette tendance est, à quelques nuances près, visible dans les deux extraits cidessous:

(1) cond40-alice: la dernière c'est un peu racaille un peu, et les autres c'est normal. le 1er, j'aime pas trop quand ça fait bourge, enfin c'est pas ça, mais c'est bizarre

(a) ent A01: (la langue utilisée dans ton quartier): c'est un petit peu la langue un petit peu mal élevée, un petit peu arabe et français

2 Les extraits du corpus de Sabatier (2004) concernant les enfants biplurilingues sont référencés avec des lettres (ex: (a)) et les extraits du corpus de Buson (en cours) concernant la variation stylistique sont référencés avec des chiffres (ex: (1)). 
Quels parallèles entre les représentations du parler plurilingue et celles du parler pluristyles chez les enfants?

On voit bien ici la prégnance du jugement social sur les variétés dans les représentations des enfants, qu'elles relèvent des langues ou des styles.

\subsubsection{Discours sur le "eux" / "nous"}

Cette distinction est bien connue dans les recherches sur le plurilinguisme depuis Gumperz et sa mise en évidence du we-code et du they-code (1982, 1989). Or, ces catégories restent pertinentes dans le domaine de la variation stylistique. On peut, en effet, voir dans les exemples qui suivent que ce discours sur le "eux" / "nous" est très présent, le "nous" correspondant aux élèves et le "eux" aux maîtresses:

(2) Laura: nous des fois on dit on dit des choses euh j(e) sais pas* on s(e) comprend quand on s(e) parle*<

(3) Abir: la maîtresse e(lle) dit il y a et moi j(e) fais il a

Enq: au lieu d'il y a Abir $\mathrm{t}(\mathrm{u})$ as fait il a<

Abir: bah ouais* bah il y a - bah (il) y a - y a

$\mathrm{XX}$ : ça s(e) voit qu(e) c'est pas des maîtresses ça s(e) voit qu(e) c'est des enfants

Enq: à quoi ça s(e) voit<

Mélissa: les maîtresses eh bah i(ls) disent pas comme ça i(ls) racontent bien: et tout et bein (il) y avait un homme< qui avait la barbe bleue < - nous on rigolait et tout

Laura: et bah moi et bah moi bein: je trouve qu'on a refait comme des maitresses pa(r)c(e) que:: pa(r)c(e) que les maîtresses i- i:: i(ls) parlent pas comme nous i(ls)::

Abir: i(ls) font un peu leur malin

Mélissa: ouais $\mathrm{p}(\mathrm{u})$ is $\mathrm{i}(\mathrm{l}) \mathrm{s}$ ont une voix bizarre tutututu (et pincée) (rires)

Abir: nous on fait XXX a(l)ors que eux i(ls) font bien

Laura: bonjour $<$ je suis $\operatorname{vot}(\mathrm{re})$ remplaçante $<\mathrm{j}(\mathrm{e})$ m'appelle naninana (intonation chantante)

Dans l'échange (3), deux élèves imitent les maîtresses représentantes du "eux" en modifiant essentiellement des éléments de prosodie ${ }^{3}$ (fortes modulations dans la courbe mélodique, variations dans la hauteur de voix, etc.). Cette même conception bipolaire de l'espace linguistique, qui induit une mise à distance des locuteurs du groupe du "dehors" ne faisant pas partie de la sphère du "nous", se retrouve dans l'enquête sur le plurilinguisme. Comme on peut le voir dans l'extrait suivant, l'enfant met en avant les usages de "l'entrenous" (langue arabe) et se positionne ainsi clairement au sein d'un groupe de référence:

(b) N00: (en quelles langues tu réponds à ton père?) français ou arabe ça dépend si on est entre nous je parlerai arabe

Cette vision dichotomique s'incarne parfois dans le clivage entre les espaces du "dedans" et du "dehors", l'espace de la famille s'opposant à celui de l'école, l'espace de la classe à celui de la cour de récréation, l'espace de l'école à celui du quartier:

(4) croiz3-lucas: il y a des phrases il parlait normal, des fois il parlait comme on parle nous,

3 On pourrait ici reprendre les termes utilisés par Barbéris (2005) et parler d'une "stylisation parodique" actualisée par une mimesis. 
dehors

(d) Y-00: quand on est dehors je parle français quand on est dedans je parle arabe

(e) A-01: alors c'est français pour quand on est dans la classe et un petit peu arabe avec les copines des fois on parle dans la cour

On constate d'ailleurs que la désignation des espaces n'est pas stable dans ces contextes: le "dehors" pouvant correspondre au "nous" des variétés informelles du quartier ou de la cour mais aussi au pôle du "eux" et des variétés formelles extra-familiales. En outre, quelques différences entre le CS et le SS sont liées à la variabilité des espaces. On recense un espace supplémentaire chez les plurilingues, l'espace étatique, empreint d'une forte résonance identitaire, comme l'illustre l'extrait (f):

(f) A-01: parce que quand on est quand quand je suis en Algérie et ben je parle plus algérien donc je me je suis comme si j'étais une VRAIE algérienne et quand je suis en France je parle vraiment français alors donc je suis comme si j'étais une Française vraiment

\subsubsection{Se distinguer, provoquer, plaisanter}

Plusieurs discours mettent clairement en évidence la valeur sociale attribuée à certaines marques, et la revendication d'une identité marginale qui peut leur être associée. Les enfants testent un code supposé crypté et de fait incompréhensible pour les extérieurs au groupe. Dans l'extrait qui suit, nous assistons à une mise en scène de ce langage du "nous", volontairement provocatrice et destinée à l'enquêteur via l'enregistreur.

(5) Ashley: bah toute la récréation tout c(e) que tu vas dire ça va ça va entendre

Laura: $\mathrm{mm}$ - même vous c(e) que vous dîtes ça entend*

Ashley: $\mathrm{X}$ sa race* regar(de) comme c'est sa race maudite*

Laura: arrê::te* $(\mathrm{XX})$ arrêtez oh::*

Le désir de choquer son auditoire peut s'accompagner d'une volonté de se distinguer de l'autre par le recours à des usages supposés emblématiques d'un groupe de locuteur de référence. Dans l'exemple ci-dessous, Abir teste avec l'enquêtrice un terme qu'elle juge appartenir au pôle du "nous" et qu'elle pense méconnu en dehors du groupe.

(6) Abir: au début en fait ça fait latche* ça fait latche et puis après ça fait plus latche

Enq: de quoi< d'avoir le truc $<$

Abir: ouais - tu comprends qu'est-c(e) que j(e) dis*<

Mélissa: (inaudible) le langage de Abir

Au delà de cette dimension cryptique, ce qui est illustré dans l'extrait (g) qui suit, c'est l'insulte rituelle, comme stratégie pour se sortir d'un mauvais pas et pour manifester une position marginale, voire pour se défendre, comme dans l'exemple (7) où ce sont les changements de styles qui sont convoqués.

(g) Mo-01: (langues utilisées avec amis du quartier) le français et euh des fois pour plaisanter le 
Quels parallèles entre les représentations du parler plurilingue et celles du parler pluristyles chez les enfants?

tunisien.

Enq: alors qu'est-ce que tu dis pour plaisanter en tunisien?

Mo-01: euh des fois on in on s'insulte mais pour rire

Enq: et tes amis qui sont en Tunisie vous vous insultez aussi en arabe?

Mo-0: non on parle en arabe mais des fois dès qu'ils m'énervent je leur euh je parle je les insulte en français (rires) comme ça ils comprennent pas

(7) barb4-linda: pour me défendre j'arrive bien mais quand je parle normalement je raccourcis pas les mots. le 3ème si on parle comme ça à un policier on sait ce qu'on va recevoir.

Il est important de souligner également le rôle de la fonction ludique, qu'on a commencé à évoquer avec les insultes. Les locuteurs utilisent certaines variantes "pour s'amuser", il y a l'idée de "moins de contrôle / de se lâcher", par opposition au caractère "sérieux" de certaines manières de parler. Cet aspect est présent dans les exemples qui suivent, et se retrouve parfois au mot près entre les deux corpus:

(8) cond32-tiphanie: c'est pour l'impression des gens, que ça peut avoir. la dernière j'aime pas trop parce que je sais pas ça fait bizarre et la 1ère j'aimais bien. moi ce serait la 2ème parce qu'elle est simple. le 3ème ça peut m'arriver quand je fais la folle.

(h) M-01: quand j'utilise l'arabe c'est pour euh pour s'amuser. et euh pour jou et euh quand y a mes copines chez moi on joue euh aux aux folles et puis on parle en arabe

(9) bea39-quentin: le 1er il est plutôt sérieux je préfère le 3ème, le 3ème il se lâche, il se laisse aller, il parle pas comme quelqu'un de sérieux, il parle à quelqu'un qu'il connaît super bien, il a pas peur de rentrer en conflit avec lui.

(i) M-01: (au Maroc avec les cousines) quand je parle en français eux ils vont ils veulent faire euh euh un peu style pour s'amuser qui savent parler en français. (le mélange) c'est un jeu pour moi

\subsection{Les fonctions / motivations du CS et du SS: convergence $v s$ divergence}

Des stratégies visant à modifier la relation interpersonnelle, à s'adapter à son interlocuteur, à entrer en connivence avec lui, à l'impliquer davantage dans la communication ont été évoquées (j) et observées (10) dans les deux corpus. La stratégie de convergence est clairement visible dans l'extrait suivant:

(j) M-01: (j'habite dans un quartier) où les jeunes sont vraiment pas polis (...) ils parlent vulgaire alors je parle vulgaire

De la même manière, l'exemple (10) met en évidence une stratégie de convergence observée chez Lilia qui passe, dans un même tour de parole, d'un style relativement informel avec ses pairs à un style d'"élève modèle" pour simuler une adresse à l'adulte.

(10) Lilia: (raconte un événement passé à une copine) on fait une course on court< d'accord* tou-tou-tou elle est tombée* - ouais - et moi j(e) te r(e)gardais dans l(e) préau - (simule l'interpellation d'un surveillant) moi je sais c(e) qui c'est passé XX i(l) m(e) dit (imite le ton de l'adulte s'adressant à un enfant) viens ici petite qu'est-c(e) qui c'est passé< j(e) (l)ui ai dit< 
(imite une petite fille innocente) on voulait faire la course sur les pou:tres< après on a couru::: < et j'ai dit allez* on accélère on a dit qu'on devait accélérer (reprend sa voix habituelle) en fait c'était tout à cause de moi hein* - Sarah $t(u)$ es une belue mai(nte)nant dans ta tête*

\section{Quelles limites à la comparaison entre CS et SS?}

La mise en perspective des deux champs de recherche s'est, nous semble-t-il, avérée pertinente: on observe en effet des traces de stratégie verbale, que ce soit dans les usages ou dans les "discours sur", à la fois dans le domaine des alternances codiques et dans celui de la souplesse stylistique. Le dernier extrait (10) est en quelque sorte emblématique de ce parallèle, puisque Lilia met en scène son propre discours au style direct. Or, cette fonction de citation est l'une des principales fonctions des changements de code chez les locuteurs qui manifestent un parler plurilingue (Deprez, 1994).

Néanmoins, il faut apporter quelques nuances à cette comparaison. Il est patent à travers notre parcours exploratoire qu'il y a une plus grande difficulté pour les enfants à se représenter et à identifier le SS par rapport au CS. Une différence importante entre les deux est en effet que:

- pour le CS: au niveau des représentations, les langues sont nommées comme des systèmes distincts. Les enfants peuvent donc concevoir le mélange de ces systèmes: des éléments qui appartiennent à un système, des éléments qui appartiennent à l'autre et des éléments de l'interlecte, du continuum, qu'ils sont nombreux à désigner sous le terme "mélange";

- pour le SS: les styles, quand ils sont nommés ${ }^{4}$, le sont comme des variétés étanches, et la notion de mélange de styles n'est jamais évoquée par les enfants, ce qui pourrait signifier que, pour eux, le mélange des styles serait plus complexe à concevoir que le mélange des langues.

Ici, il est alors remarquable que la conception non experte rejoint la conception du linguiste: il n'y a en effet pas de mélange de styles à proprement parler, juste, tout au plus, une congruence de traits dans le discours, où la simple occurrence d'un trait saillant susceptible de porter des connotations sociales très fortes, peut induire, en réception, un changement de style pour un auditeur donné, dans un contexte donné (voir les indices de contextualisation chez Gumperz, 1982).

En réalité, ce n'est pas un parallèle qu'il faudrait faire entre CS et SS, puisque l'on parle d'une même réalité chez le locuteur: le répertoire plurilingue n'est pas constitué d'une langue + d'une seconde (ou plus) langue + du mélange des deux, mais bien d'un continuum intégrant les langues, leur mélange ET le continuum stylistique inhérent à chacun des éléments, le tout sur le même niveau, et non sur deux niveaux parallèles qui par définition ne se rencontreraient jamais. Le répertoire monolingue ne serait alors en quelque sorte

4 Très peu d'élèves y font référence spontanément (Buson, en cours): par exemple, seuls 2 enquêtés sur 196 emploient le terme de "langage soutenu", pourtant fréquemment utilisé dans les manuels scolaires. 
Quels parallèles entre les représentations du parler plurilingue et celles du parler pluristyles chez les enfants? qu'un cas particulier du répertoire plurilingue. 


\section{Bibliographie}

Barbéris, Jeanne-Marie (2005): Le processus dialogique dans les phénomènes de reprise en écho. In: Bres, Jacques / Haillet, Pierre-Patrick / Mellet, Sylvie / Nölke, Hans / Rosier, Laurence (edd.): Dialogisme et polyphonie. Approches linguistiques. Bruxelles: De Bøck-Duculot, 157-172.

Bell, Allan (2001): Back in style: reworking audience design. In: Eckert, Penelope and Rickford, John R. (edd.): Style and sociolinguistic variation. Cambridge: Cambridge University Press, 139-169.

Bernstein, Basil (1975): Langage et classes sociales. Codes socio-linguistiques et contrôle social. Paris: Les éditions de minuit.

Billiez, Jacqueline (1997): Bilinguisme, immigration, variation: regards sociolinguistiques. Dossier H.D.R., Université Stendhal Grenoble 3.

Buson, Laurence (en cours): La variation stylistique chez les enfants de 9 à 12 ans. Représentations et pratiques. Thèse préparée sous la dir. de Jacqueline Billiez, Université Stendhal Grenoble 3.

Buson, Laurence (2008): La variation stylistique chez les enfants de 10/11 ans: une étude exploratoire en contexte français. In: Abecassis, Michaël / Ayosso, Laure / Vialleton, Elodie (edd.): Le Français parlé au XXIème siècle. Normes et variations géographiques et sociales. Paris: L'Harmattan, 203-222.

Deprez, Christine (1994): Les enfants bilingues: langues et familles. Paris: Didier.

Gadet, Françoise (2006): Le locuteur comme champ de bataille (version provisoire). Archives de la journée de l'I.U.F. du 12 mai 2006, en ligne, http://www.unice.fr/ChaireIUF-Nicolai.

Gadet, Françoise / Varro, Gabrielle (edd.) (2006): Le 'scandale' du bilinguisme. Langues en contact et plurilinguismes. Langage et Société 116. Paris: Maison des sciences de l'homme.

Gadet, Françoise/Tyne, Henry (2007): Le style en sociolinguistique: ce que nous apprend l'acquisition. In: Rabatel, Alain / Petitjean, André (edd.): Questions de style. Metz: Cresef, 91-99.

Gumperz, John J. (1964): Linguistic and social interactions in two communities. In: Gumperz, John J. / Hymes, Dell (edd.) The ethnography of communication. American Anthropologist 66(6), 137153.

Gumperz, John J. (1982): Discourse strategies. Cambridge: Cambridge University Press.

Gumperz, John J. (1989): Sociolinguistique interactionnelle. Une approche interprétative. Paris: L'Harmattan.

Labov, William (1972): Sociolinguistic patterns. Oxford: Basil Blackwell.

Labov, William (1976): Sociolinguistique. Paris: Les éditions de minuit.

Lüdi, Georges / Py, Bernard (32003): Etre bilingue. Berne: Peter Lang.

Sabatier, Cécile (2004): Rôle de l'école dans le développement et la construction du plurilinguisme chez des enfants issus de la migration maghrébine en France. Thèse présentée sous la dir. de Jacqueline Billiez, Université Stendhal Grenoble 3.

Schilling-Estes, Natalie (2002): Investigating stylistic variation. In: Chambers, J.K. / Trudgill, Peter et al. (edd.) The handbook of language variation and change, 375-401. 